\title{
Improved OD and OC Fragmentation using Deep Learning for the Progression Recognition of Glaucoma
}

\section{Shanmugam P}

Adhiparasakthi Engineering College

Raja J ( $\nabla$ raja.stj@gmail.com )

Adhiparasakthi Engineering College

Pitchai R

B V Raju Institute of Technology

\section{Research Article}

Keywords: Deep learning, Glaucoma, Optic disc, Optic cup, Multi-label segmentation U-net, RF Classifier

Posted Date: June 3rd, 2021

DOI: https://doi.org/10.21203/rs.3.rs-490801/v1

License: (c) (i) This work is licensed under a Creative Commons Attribution 4.0 International License.

Read Full License 


\title{
Improved OD and OC Fragmentation using Deep Learning for the Progression Recognition of Glaucoma
}

\author{
${ }^{1}$ Shanmugam P, ${ }^{2 *}$ Raja J, ${ }^{3}$ Pitchai R \\ ${ }^{1}$ Assisttant Professor, Department of IT, Adhiparasakthi Engineering College, \\ Melmaruvathur, Tamilnadu, India. \\ ${ }^{2}$ Professor, Department of ECE, Adhiparasakthi Engineering College, \\ Melmaruvathur, Tamilnadu, India. \\ ${ }^{3}$ Associate Professor, Department of CSE, B V Raju Institute of Technology, Narsapur, Telangana, India. \\ ${ }^{1}$ shanmugam.shanvish2003@gmail.com, ${ }^{* 2}$ raja.stj@gmail.com, ${ }^{3}$ pitchrks1984@gmail.com
}

\begin{abstract}
Glaucoma is one of the most hazardous diseases, proceeding to impact and burden an extensive bit of our general population. Appropriately, the initial stage of identification of glaucoma is significant to prevent the permanent vision misfortune. The CDR is the important factor for glaucoma recognition. The precise fragmentation of optic disc and cup is yet an evolving issue. Most of the segmentation based glaucoma recognition methods depends on the handcrafted features. It affects the overall performance of the glaucoma recognition. To resolve this issue, an efficient deep learning based optic cup and disc segmentation using technique multi-label segmentation Au-net has been developed in this paper. The proposed method focusing on the optic cup-to-disc ratio for the recognition of glaucoma, which may be the best system for building a capable, energetic, and accurate structure for glaucoma analysis. This system has been simulated on DRISHTI datasets. The exploratory outcomes indicates the proposed strategy performs well to the best with state-of-the-art methodologies accomplishing a 99\% of Accuracy, $88 \%$ of Sensitivity and $95.5 \%$ of Specificity on the DRISHTI GS1 dataset individually.
\end{abstract}

Keywords: Deep learning, Glaucoma, Optic disc, Optic cup, Multi-label segmentation U-net, RF Classifier

\section{Introduction}

Glaucoma is the common reasons for visual deficiency, it is anticipated to influence about 80 million individuals by 2020 [1]. It is a persistent eye infection that prompts vision misfortune, in which the optic nerve is dynamically harmed. It is otherwise named as silent thief of sight, since the symptoms possibly occur in advance stage. The impact of the disease could be determined from the prediction that the populace influenced from glaucoma may expand from 80 million of in 2020 to 111.8 million in 2040 [2]. The individuals among 40 to 64 years old are encouraged to complete 
their eye test once in every 2 to 4 years [3]. These eye test has been taken by the experts, it will increase the possibility of human error. The Computer-Aided Diagnostic frameworks could assist with decreasing the flaws and may help the medical care specialists in an unprejudiced screening of patients [4-5].

Even though glaucoma can't be relieved, the progression of glaucoma can be eased by proper eye treatment. For early recognition of glaucoma effective fundus images are required. Different parameters such as CDR[6], disc measurement [7], ISNT rule [8], and Peripapillary atrophy [9] are used to recognize the glaucoma. CDR is one of the commonly utilized parameter by experts [10]. It is determined by taking the proportion of Vertical Cup Width (VCD) to Vertical disc diameter (VDD), as indicated in Fig. 1. The manual assessment of CDR is tedious and exorbitant, and it is not reasonable for large-scale screening. Extricating the region of interest (ROI) takes lesser time to measure the optic cup (OC) and optic disc (OD) region [11].

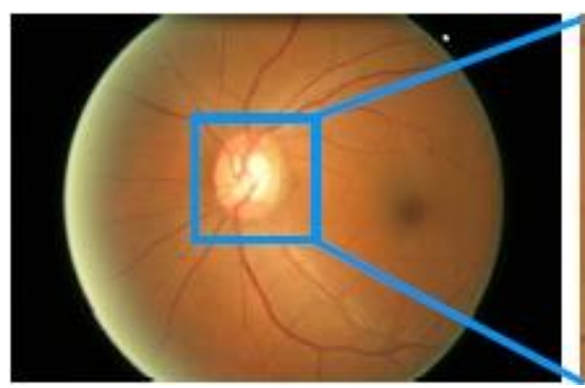

a) Fundus Image

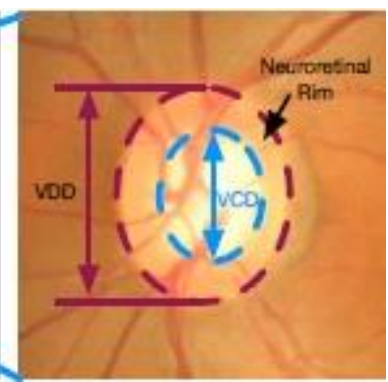

b) Normal

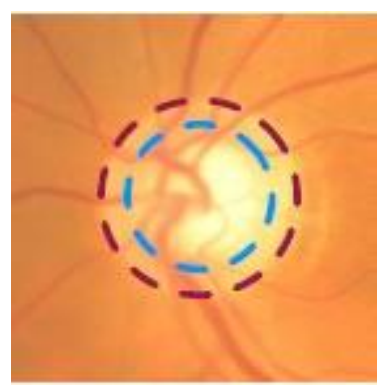

c) Glaucoma

Fig 1: Normal and Glaucoma Fundus images

For glaucoma recognition, the illness pattern in retinal fundus image is unpredictable and covered up, which is not similar to the natural images. The illness pattern could be observed by the clinical experts. The Deep Learning (DL) structures are developed by using linear/non-linear conversions of information, it is most widely used for glaucoma recognition [12]. Here, we are persuaded to develop a DL based methodology for extracting the characteristics for accurate glaucoma recognition.

This paper is further coordinated as follows; Section 2 briefly explain the proposed methodology using multi-label segmentation using Au-net; Section 3 presents the simulation outcomes and analysis; and lastly, conclusion of this work has been given in Section 4. 


\section{Methodology}

The block diagram of proposed methodology used to recognize the glaucoma has been portrayed in fig 2. The multi-label segmentation Au-Net is a one-stage framework [13], used to fragment the OD and OC from the retinal images. In this network, the multi-scale layer assembles an image pyramid to give multi-level inputs. Here, four different streams are utilized. The first stream act as the classifier on the fundus images straightforwardly. The subsequent stream is a fragmentation guided structure, it restricts the OD locale from the retinal imageries and inserts the OD fragmentation portrayal to recognize glaucoma. The third steam deals with local OD locale to anticipate the likelihood from the OD locale. The fourth stream concentrated on OD locale with polar transformation, it develops the OD and OC framework with the geometry operation and increases the efficiency of segmentation. At last, each output from all the different streams are melded to yield the result. At this stream, a multi-label loss function is used to ensure fragmenting the OD and OC. This proposed net creates the OD and OC fragmentation maps, then determines the CDR dependent on fragmented OD and OC to find the risk level of glaucoma. In the multi-label segmentation Au-net, the OD locale is initially confined by utilizing the automatic OD recognition technique, and afterward the input image is converted to polar co-ordinate system. Then this image is given to the Au-net to foresee the multi-label probability maps for OD and OC locale. At long last, the converse polar transformation recuperates the fragmentation map back to the Cartesian coordinate system.

\subsection{Multi-level Au-Net}

The Au-Net [14] has been developed from of U-net. The multi-label segmentation Au-net comprises of the encoder and decoder whose operation is same as U-net. These encoder and decoder generate the feature map. The skip association transfers the feature map from encoder path and connects them to up-sampled decoder feature maps. The multi-scale info is used to increase the efficiency of segmentation $[15,16]$. The multi-label segmentation Au-net utilizes the normal pooling layer to down-sample the image normally and build a multi-scale contribution to the encoder path. The final decoder output is given to the classifier network. This classifier uses convolutional layer with Sigmoidal initiation to yield the likellihood map. Here, the multi-label fragmentation network gives $K$ channel probability map. 


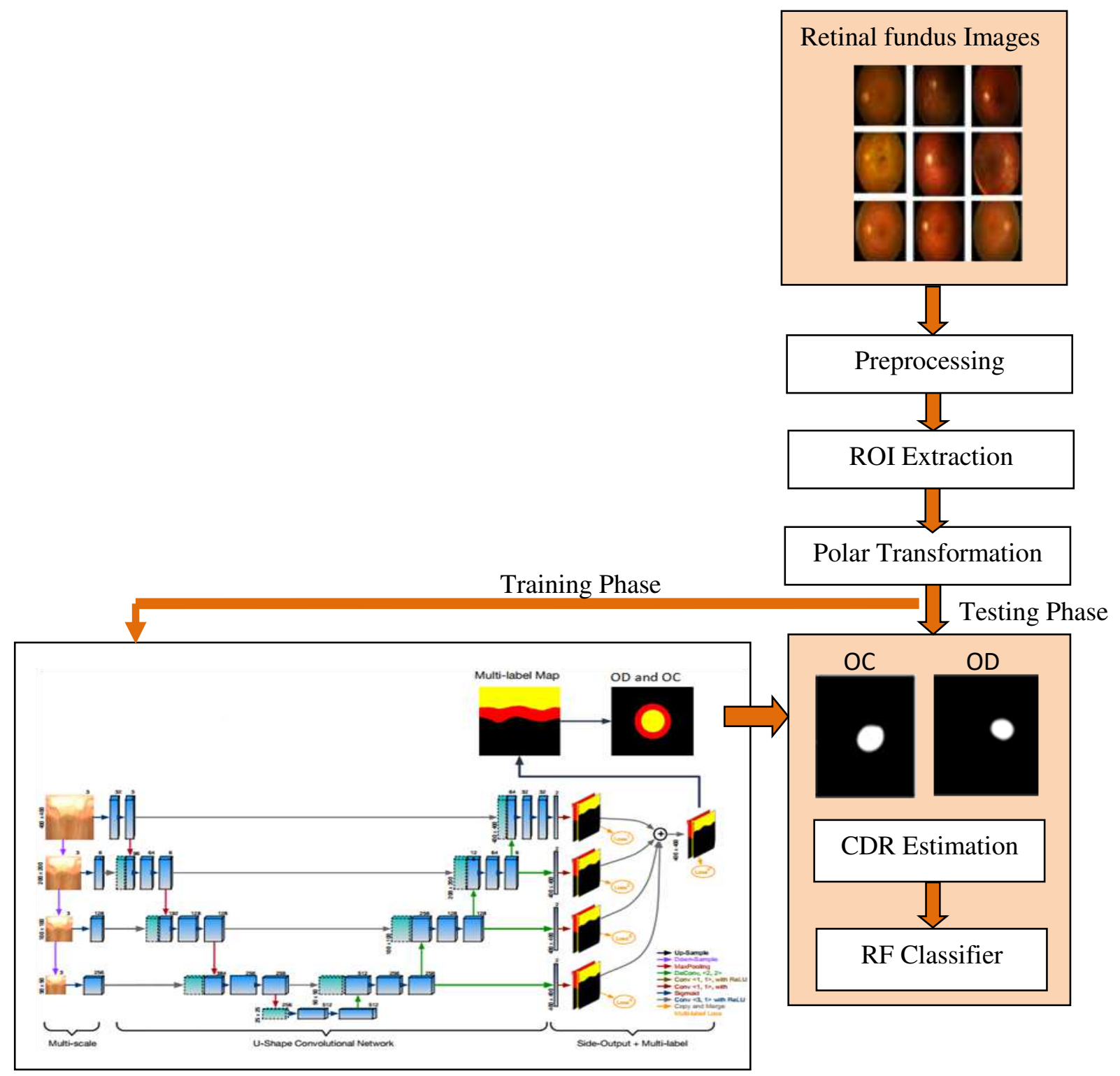

Fig 2: Block diagram of OD and OC segmentation using Multi-label segmentation Au-net

\subsection{Side-output Layer}

This segmentation technique is used for separating people into groups based on their labels Au-net employs a side layer that performs the initial classification to include a local output chart [17]. Let $W$ represent the coefficients of regular convolutional layers, and let $M$ represent the weights of all side-output layers, where $\mathrm{w}=\left(\mathrm{w}^{(1)} \ldots \ldots \ldots . \mathrm{w}^{(M)}\right)$. The side-output layer's objective feature is as follows:

$$
\mathrm{L}_{\text {side }}(\mathrm{W}, \mathrm{w})=\sum_{\mathrm{m}=1}^{\mathrm{M}} \alpha_{\mathrm{m}} \mathrm{L}_{\text {side }}^{(\mathrm{m})}\left(\mathrm{W}, \mathrm{w}^{(\mathrm{m})}\right)
$$


Here $a_{m}$ represents the multi-label loss function $\left(a_{m}=0.25\right)$, and $L_{\text {side }}^{(m)}($, ) represents the loss function. The merits of side-output layer are: a) it could be perceived the association among the loss and early layer. and b) multi-scale output unification may provide better performance.

\subsection{Multi-label Loss Function}

The pixel fragmentation problems is conceived as a multi-label issue in the proposed multilabel network. The current segmentation approach is associated with a multi-class environment, in which each instance is assigned to a unique label. On the other hand, Multi-label uses a separate binary classifier for every class and assigns each instance to several binary labels. The OD locale overlays the OC pixels, particularly for OD and OC fragmentation, which implies that a pixel labeled as cup often marked as disc. Furthermore, in glaucoma situations, the disc pixels omitted cup area is shaped like a ring, making the disc label highly unbalanced in comparison to the context label in a multi-class environment. As a result, the multi-label approach, which treats OD and OC as two separate areas, is better suited to dealing with these problems. A multi-label loss function in Au-Net, and it is described as:

$$
L_{s}=1-\sum_{k}^{K} \frac{2 w_{k} \sum_{i}^{N} P_{(k, i)} g_{(k, i)}}{\sum_{i}^{N} P_{(k, i)}^{2}+\sum_{i}^{N} g_{(k, i)}^{2}}
$$

Here $N$ represents the pixel size, $g_{(k, i)} \in\{0,1\}$ and $p_{(k, i)}\{0,1\}$ represents the ground truth and the expected likelihood for class $k$. The class weights are $\sum_{k} w_{k}=1$. The pixel are labeled as OD or OC separately using the multi-label configuration. As a result, there is no imbalance between OD and OC. The trade-off weight $w_{k}$ in Eq. (2) is used to regulate the contribution of OD and OC. In this glaucoma screening, $w_{k}=0.5$ is used. The gradient can be obtained by differentiating the multi-label loss function Ls.

$$
\frac{\partial L_{s}}{\partial P_{(k, i)}}=\sum_{k}^{K} 2 w_{k}\left[-\frac{g_{(k, i)}}{\sum_{i}^{N} P_{(k, i)}^{2}+\sum_{i}^{N} g_{(k, i)}^{2}}+\frac{2 P_{(k, i)} \sum_{i}^{N} P_{(k, i)} g_{(k, i)}}{\left(\sum_{i}^{N} P_{(k, i)}^{2}+\sum_{i}^{N} g_{(k, i)}^{2}\right)^{2}}\right]
$$

\subsection{Polar Transformation}

In the multi-label segmentation Au-Net, the pixel-wise polar transformation has been utilized. It can be expressed as follows:

$$
\left\{\begin{array}{l}
u=u_{o}+r \cos (\theta+\varnothing) \\
v=v_{o}+r \sin (\theta+\varnothing)
\end{array}\right.
$$

and the inverse transformation is given as: 


$$
\left\{\begin{array}{c}
r=\sqrt{\left(u-u_{o}\right)^{2}+\left(v-v_{o}\right)^{2}} \\
\theta=\tan ^{-1}\left(\frac{v-v_{o}}{u-u_{o}}\right)-\emptyset
\end{array}\right.
$$

The polar image's height and width are determined by the polar radius $r$ and the discretization $2 p=s$, here $s$ represents the stride. It has the following properties:

Geometric Restriction: The OC region must be inside the OD region. In Cartesian co-ordinate system, it is very difficult to implement the $\mathrm{OC}$ and $\mathrm{OD}$ region. But, in polar transformation, the OC,OD and background locale are separated in a layered structure $[18,19]$.

Analogous Augmentation: The polar transition is equivalent to data augmentation since it is a pixel-wise projection. In the augmentation process, increasing the transition radius is simiar to the scaling factor. As a result, data augmentation for DL could be achieved effectively.

Cup Proportion Balancing: The distribution of OC and context pixels in the fundus image is severely skewed. The area of optic cup is always lesser when compared with ROI region. Usually, the cup region occupies about $4 \%$ of the total area. This highly unbalanced ratio quickly contributes to prejudice and overfitting in deep model training. The polar transformation flattens the image centred on the OD nucleus, allowing interpolation to expand the cup area and raise the OC proportion. In this paper, the ratio of cup region over the ROI improves to 23.4 percent, this helps to prevent overfitting during model training and boost $\mathrm{OC}$ fragmentation even more.

\subsection{Random Forest Glaucoma Classification from Morphometric Attributes}

The third stage is a Random Forest Classifier that collects the 19 MAs and categorizes them into two probable classes such as healthy, or glaucoma. The functioning of this algorithm is similar to the decision tree algorithm. This algorithm collects all predictions from decision trees and finds the best solution. Each tree depends upon the random feature set which is described in Fig.3. 


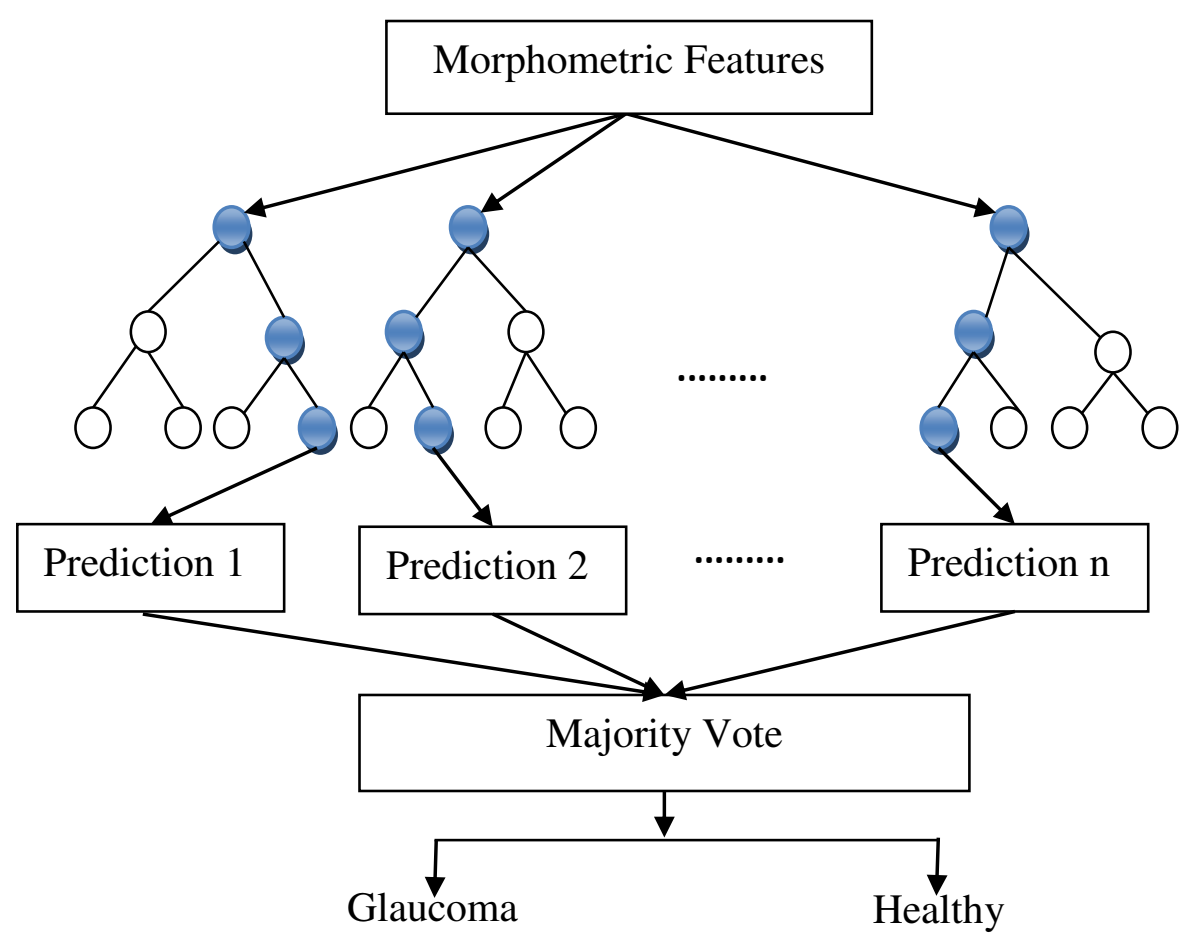

Fig 3: Random Forest Classification

The Gini index is a proportion of prediction intensity of factors in regression, depends on the impurity reduction procedure. It can be utilized to rank the significance of attributes for an order issue. It is non-parametric and it doesn't depend on information having a place with a specific sort of conveyance. For a binary, Gini index is determined as pursues:

$$
\operatorname{Gini}(x)=1-\sum_{j=1}^{2}\left(P_{j}\right)^{2}
$$

Where $p_{j}$ represents the occurrence of class $j$ in the hub $n$. For dividing a binary node in the most ideal manner, the enhancement in the Gini index must be boosted. The lower value of the Gini index implies that a specific indicator plays a significant role in categorizing the data into the two classes.

\section{Segmentation Evaluation}

Here, the performance of OD and OC fragmentation using multi-label Au-net has been evaluated. The public dataset DRISHTI-GS1 [20,21] has been used for this experiment. This dataset has 101 images. Among this 50 images have been utilized for training and 51 fundus images have been used for testing. Here, all the 50 images are marked by 5 glaucoma domain experts. The polar transformation radius is set to 400 and the learning rate is 0.0001 , the size of the converted imageries are $400 \times 400$. The threshold value of 0.5 has been used to get the binary segmented region of OC and OD respectively. 

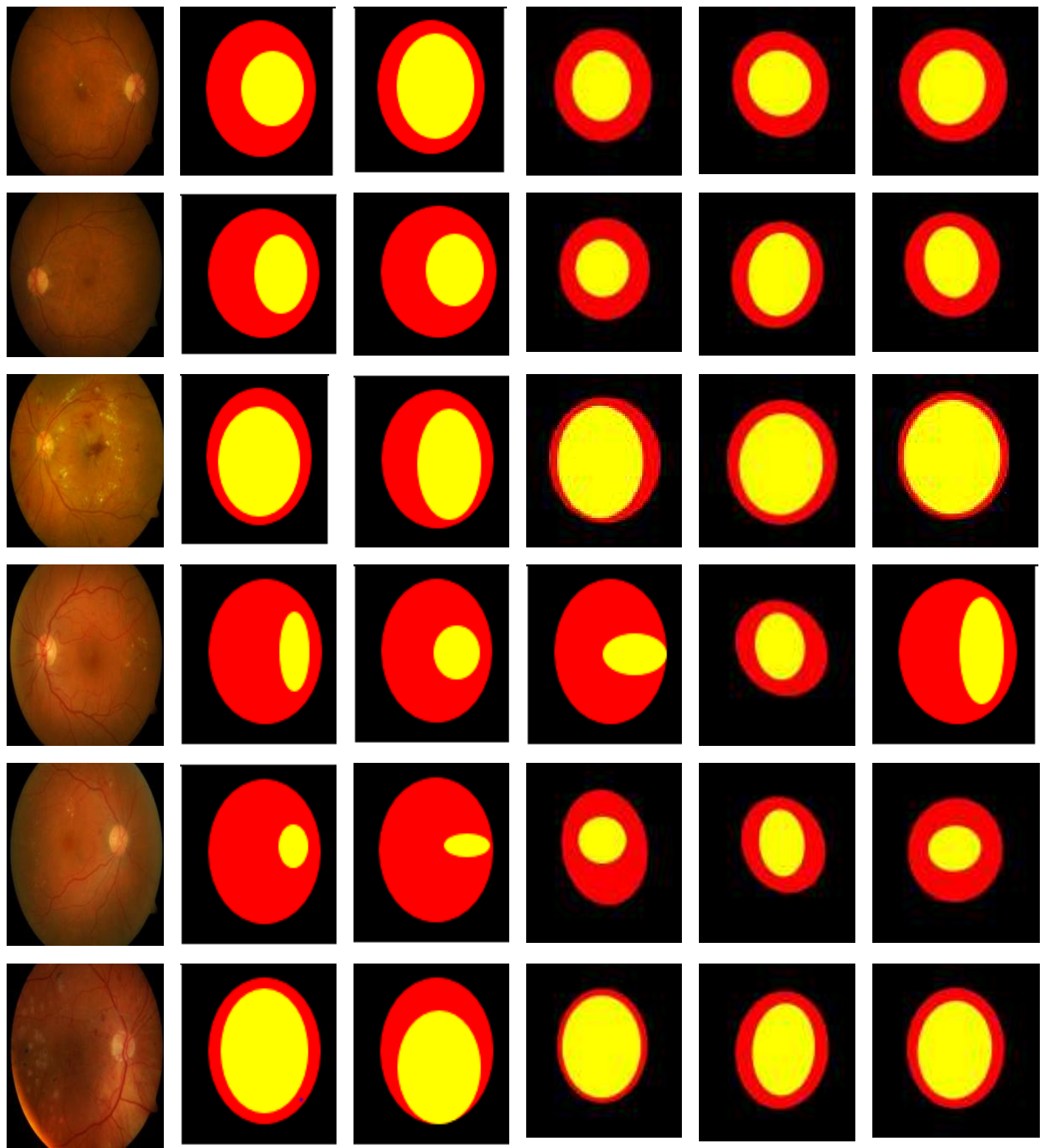

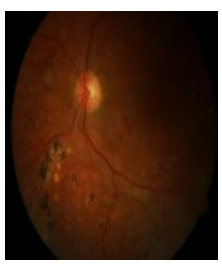

(a)

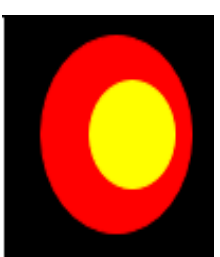

(b)

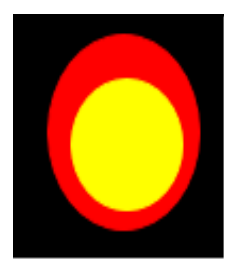

(c)

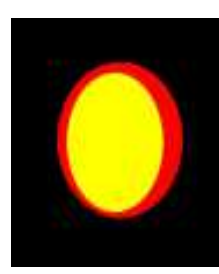

(d)

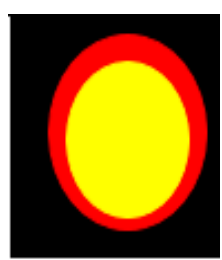

(e)

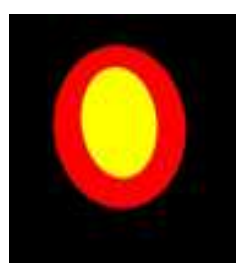

(f)

Fig 4: Simulation results a) original fundus image b) Original U-Net c) Full-Deformable U-Net d)

Deformable U-Net e) Au-Net and g) proposed method

Fig 4. provides some graphic representations of the fragmentation findings. In the above figure, the yellow and red locale represents the OC and OD separately. The simulation results of proposed methodology has been compared with various segmentation methods such as Original UNet, Full-Deformable U-Net, Deformable U-Net and Au-Net. The performance of the above 
mentioned methods are evaluated using different parameters such as Sensitivity, Specificity and Accuracy. They are discussed as follows,

\subsection{Sensitivity}

It is calculated by taking the proportion among the correct positive predictions and the total number of positives. The best sensitivity is 1 , whereas, the worst case + is 0 . It is expresses as follows [22],

$$
\text { Recall }=\frac{T P}{T P+F N}
$$

\subsection{Specificity}

Specificity reflects the true negative rate of class. It is the ratio of correct negative predictions and the total number of negatives. The best specificity is 1 , whereas the worst is 0 .It is expressed as follows,

$$
\text { Specificity }=\frac{T N}{T N+F P}
$$

\subsection{Accuracy}

Accuracy is the ratio of the total number of predictions that are correct. 99\% accuracy can be excellent. It is expressed as follows [22],

$$
\text { Accuracy }=\frac{T P+T N}{T P+T N+F P+F N}
$$

In the above equations $(7,8,9), \mathrm{TP}$ and $\mathrm{TN}$ represents the number of true positive and true negative respectively, and FP and FN represents the false positive and false negative respectivey

Table.1 Parameter evauation for DRISHTI-GS dataset for OD and OC Segmentation

\begin{tabular}{|l|c|c|c|c|c|c|}
\hline \multirow{2}{*}{ Methods } & \multicolumn{3}{c|}{ Optic Cup } & \multicolumn{3}{c|}{ Optic Disc } \\
\cline { 2 - 7 } & Acc & SN & SP & Acc & SN & SP \\
\hline Multi-label segmentation Au-net & 99 & 87 & 96 & 99 & 89 & 95 \\
\hline Au-Net & 99 & 86 & 95 & 99 & 87 & 92 \\
\hline Deformable U-Net & 98 & 84 & 92 & 99 & 85 & 89 \\
\hline Full-Deformable U-Net & 95 & 84 & 92 & 96 & 83 & 95 \\
\hline Original U-Net & 84 & 82 & 85 & 86 & 81 & 81 \\
\hline
\end{tabular}




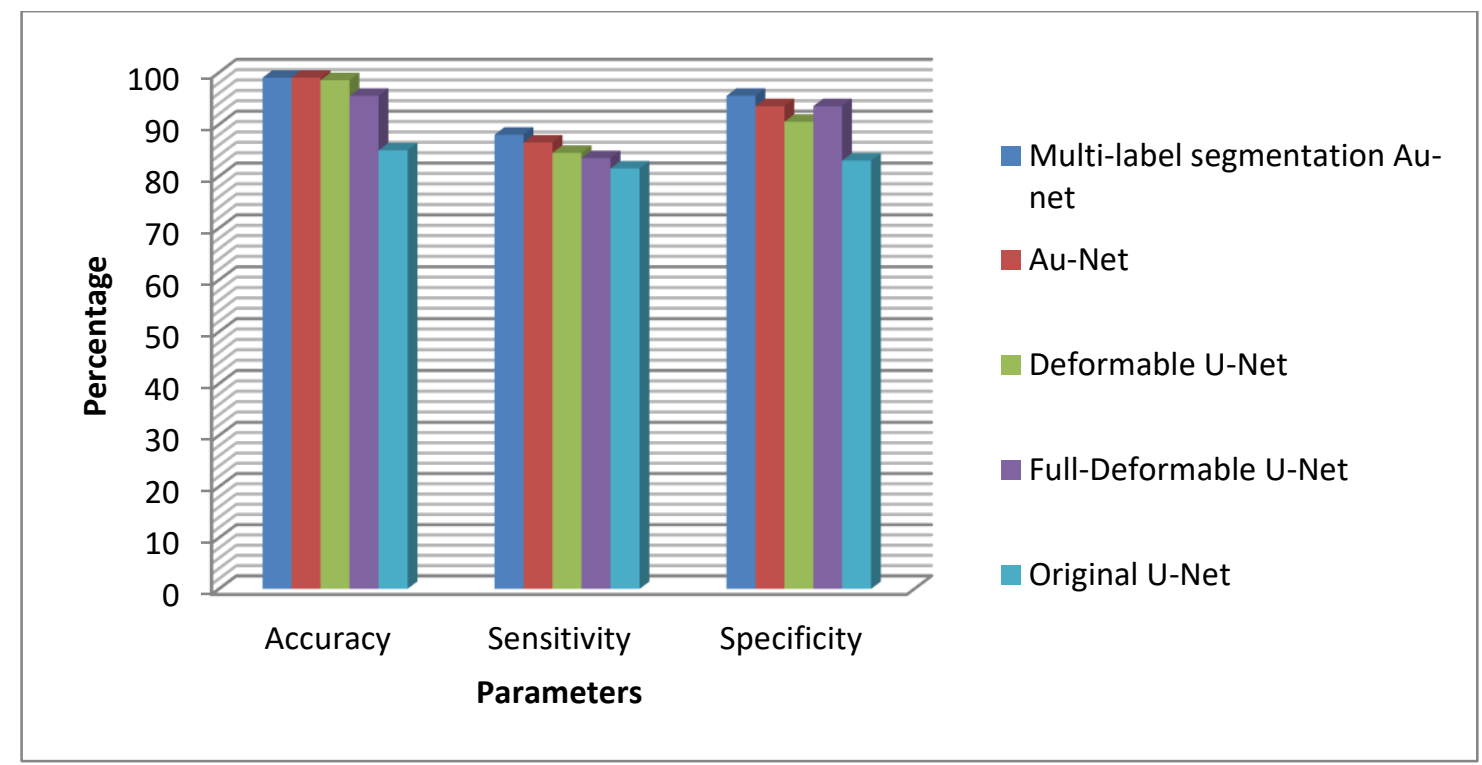

Fig 5: Comparative analysis of Proposed method with different segmentation methodoogies

The analysis of the Multi-label segmentation Au-Net with the state-of-arts methods is shown in Fig.5. It is evident that the efficiency of the Au-Net methodology has enhanced the glaucoma detection when correlated with existing methodologies.

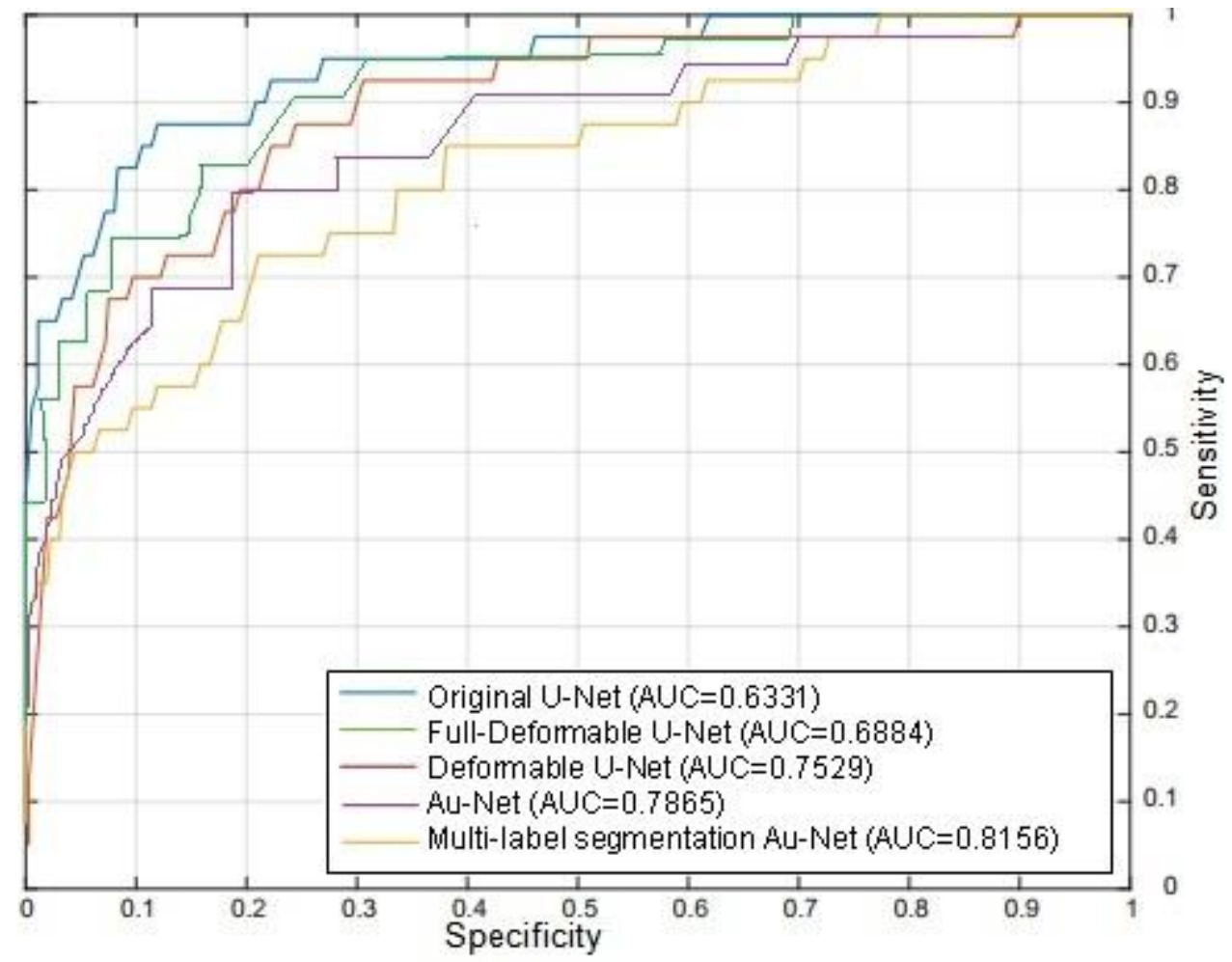

This paper additionally compute the intraocular pressure and the simulation outcomes are shown in Fig 6. From the table, we can notice that, the Au-Net and Multi-label segmentation AuNet obtains higher score than the Full-Deformable U-Net and Original U-Net. This shows that the multi-label inputs and single side output layers are helpful in layer training to get better performance. 
The merit of this proposed methodology is polar transformation, it solves the imbalance issues occurred during OC and OD segmentation. This balanced region avoid the over-fitting and helps to improve the segmentation performance.

\section{Conclusion}

In this paper, we have discussed and analyzed the performance of multi-label fragmentation $\mathrm{Au}-\mathrm{Net}$ with other existing methodologies. The multi-label segmentation $\mathrm{Au}-\mathrm{Net}$ integrates four deep streams on different levels and recognize glaucoma from the retinal imageries. The design of this Au-Net is comprises of three fundamental parts. The first is a multi-scale U-shape convolutional network, significant features are obtained from this network. Then, the side-output layer gives deeply supervision. Finally, the loss function is used to fragment OD and OC locale together. For accurate segmentation, it additionally utilizes a polar transformation to convert the input image into polar transformed image. The experiment has been performed on the DRISHTI-GS1 glaucoma dataset and it yields effective performances. This proposed method can be used to diagnose eye disease in automated retinal image processing systems.

\section{Compliance with Ethical Standards}

* Disclosure of potential conflicts of interest:

(In case of Funding) Funding: Funding is not applicable.

Conflict of Interest: No Conflict of Interest

* Research involving human participants and/or animals:

Ethical approval: This article does not contain any studies with human participants or animals performed by any of the authors.

*Informed consent: Not Applicable

\section{References}

1. Quigley, H.A., Broman, A.T.: The number of people with glaucoma worldwide in 2010 and 2020. In: Ophthalmol 2006

2. Tham YC, Li X, Wong TY, Quigley HA, Aung T, Cheng CY (2014) Global prevalence of glaucoma and projections of glaucoma burden through 2040: a systematic review and meta-analysis. Ophthalmology 121(11):2081-2091

3. Glaucoma Research Foundation, "Five common glaucoma tests", https://www.glaucoma.org/glaucoma/diagnostic-tests.php, 2017 
4. Hagiwara Y, Koh JEW, Tan JH, Bhandary SV, Laude A, Ciaccio EJ, Tong L, Rajendra Acharya U (2018) Computer-aided diagnosis of Glaucoma using fundus images: a review, Computer Methods and Programs in Biomedicine

5. Quigley HA, Green WR (1979) The histology of human glaucoma cupping and optic nerve damage: clinical correlation in 21 eyes. Ophthalmology 86(10):1803-1830

6. Damms, T., Dannheim, F.: Sensitivity and specificity of optic disc parameters in chronic glaucoma. In: Invest. Ophth. Vis. Sci. 1993

7. Michael, D., Hancox, O.D.: Optic disc size, an important consideration in the glaucoma evaluation. In: Clinical Eye and Vision Care 1999

8. Harizman, N., Oliveira, C., Chiang, A., Tello, C., Marmor, M., Ritch, R., Liebmann, J.M.: The isnt rule and differentiation of normal from glaucomatous eyes. In: Arch Ophthalmol 2006

9. Jonas, J.B., Fernandez, M.C., Naumann, G.O.: Glaucomatous parapapillary atrophy occurrence and correlations. In:

Arch

Ophthalmol 1992

10. Jonas, J.B., Bergua, A., SchmitzValckenberg, P., Papastathopoulos, K.I., Budde, W.M.: Ranking of optic disc variables for detection of glaucomatous optic nerve damage. Invest. Ophthalmol. Vis. Sci. 41(7), 1764-1773 (2000)

11. Cheng, J., Li, Z., Gu, Z., Fu, H., Wong, D.W.K., Liu, J.: Structure-preserving Guided Retinal Image Filtering and Its Application for Optic Disc Analysis. IEEE Transactions on Medical Imaging 37(11), 2536-2546 (2018)

12. Muhammad Naseer Bajwa1, Muhammad Imran Malik, Shoaib Ahmed Siddiqui1, Andreas Dengel, Faisal Shafait, Wolfgang Neumeier and Sheraz Ahmed, "Two-stage framework for optic disc localization and glaucoma classification in retinal fundus images using deep learning”, BMC Medical Informatics and Decision Making (2019) 19:136, 1-17.

13. Fu, H., Cheng, J., Xu, Y., Wong, D., Liu, J., Cao, X.: Joint Optic Disc and Cup Segmentation Based on Multi-label Deep Network and Polar Transformation. IEEE Transactions on Medical Imaging 37(7), 1597-1605 (2018)

14. Li, G., Yu, Y.: Visual saliency detection based on multiscale deep cnn features. IEEE Trans. Image Process. 25(11), 5012-5024 (2016)

15. Liu, Y., Cheng, M.M., Hu, X., Wang, K., Bai, X.: Richer Convolutional Features for Edge Detection. In: Proc. CVPR (2017)

16. Ronneberger, O., Fischer, P., Brox, T.: U-Net: Convolutional Networks for Biomedical Image Segmentation. In: Proc. MICCAI, pp. 234-241 (2015) 
17. Lee, C., Xie, S., Gallagher, P., Zhang, Z., Tu, Z.: Deeply-supervised nets. In: International Conference on Artificial Intelligence and Statistics (2015)

18. Lang, A., Carass, A., Hauser, M., Sotirchos, E.S., Calabresi, P.A., Ying, H.S., Prince, J.L.: Retinal layer segmentation of macular oct images using boundary classification. Biomed. Opt. Express 4(7), 1133-1152 (2013)

19. Dufour, P.a., Ceklic, L., Abdillahi, H., Schroder, S., De Dzanet, S., Wolf-Schnurrbusch, U., "Kowal, J.: Graph-Based Multi-Surface Segmentation of OCT Data Using Trained Hard and Soft Constraints. IEEE Trans. Med. Imag. 32(3), 531-543 (2013)

20. Aquino A, Gegúndez-Arias ME, Marín D. Detecting the optic disc boundary in digital fundus images using morphological, edge detection, and feature extraction techniques. IEEE Trans Med Imaging. 2010;29(11):1860-9

21. Partha Sarathi Mangipud i, Hari Mohan Pandey, Ankur Choudhary, "Improved optic disc and cup segmentation in Glaucomatic images using deep learning architecture" Multimedia Tools and Applications, 2021, 1-21. https://doi.org/10.1007/s11042-020-10430-6

22. Kalaiyarasi Murugesan, Perumal Balasubramani, Pallikonda Rajasekaran Murugan, Saravanan Sankaranarayanan, "Color-Based SAR Image Segmentation Using HSV+FKM clustering for Estimating the Deforestation Rate of LBA-ECO LC-14 Modeled Deforestation Scenarios, Amazon Basin: 2002-2050”, Arabian Journal of Geosciences, 14, 777 (2021). https://doi.org/10.1007/s12517021-07069-4 
Figures

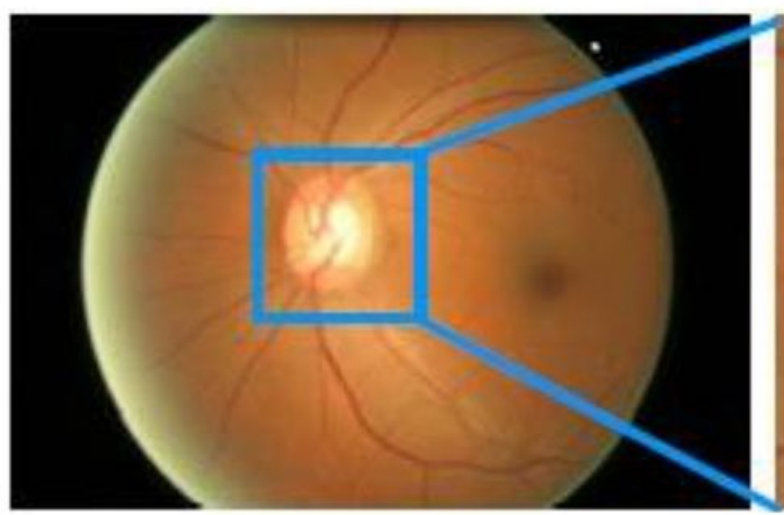

a) Fundus Image

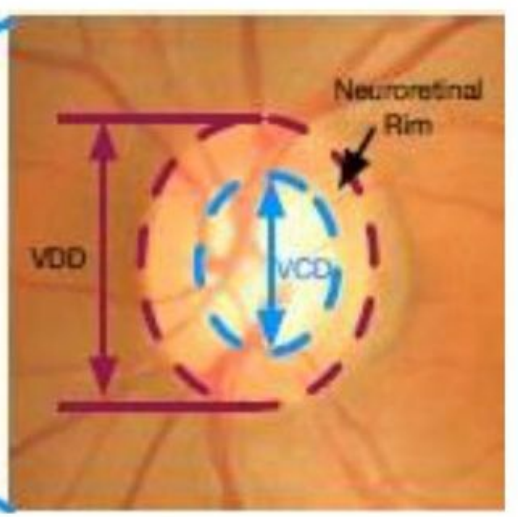

b) Normal

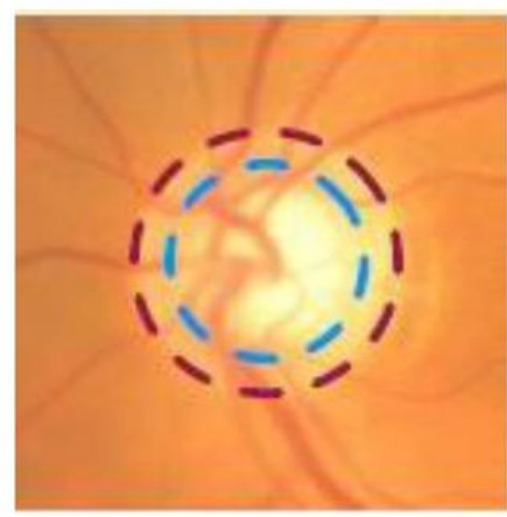

c) Glaucoma

Figure 1

Normal and Glaucoma Fundus images 


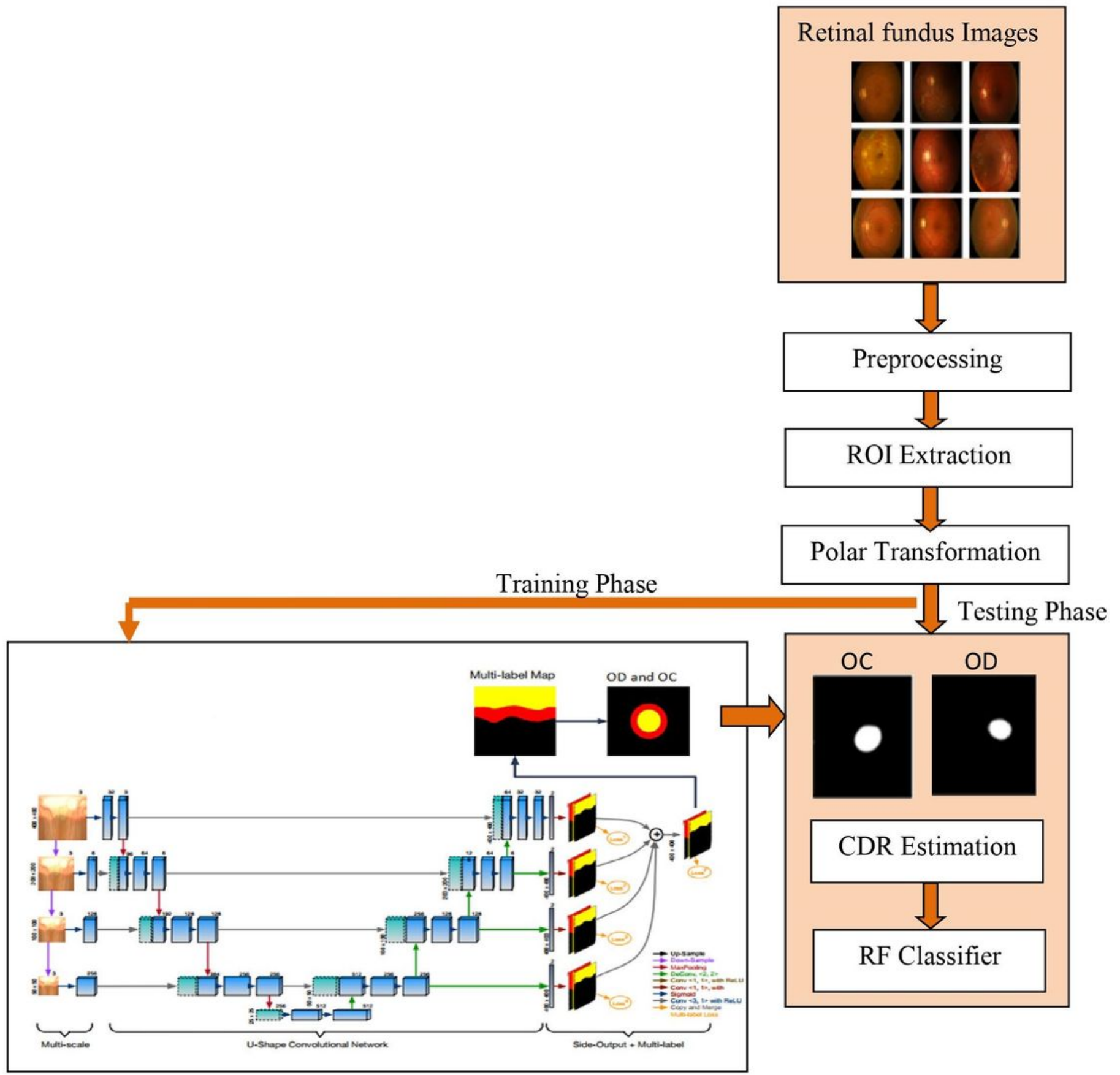

Figure 2

Block diagram of OD and OC segmentation using Multi-label segmentation Au-net 


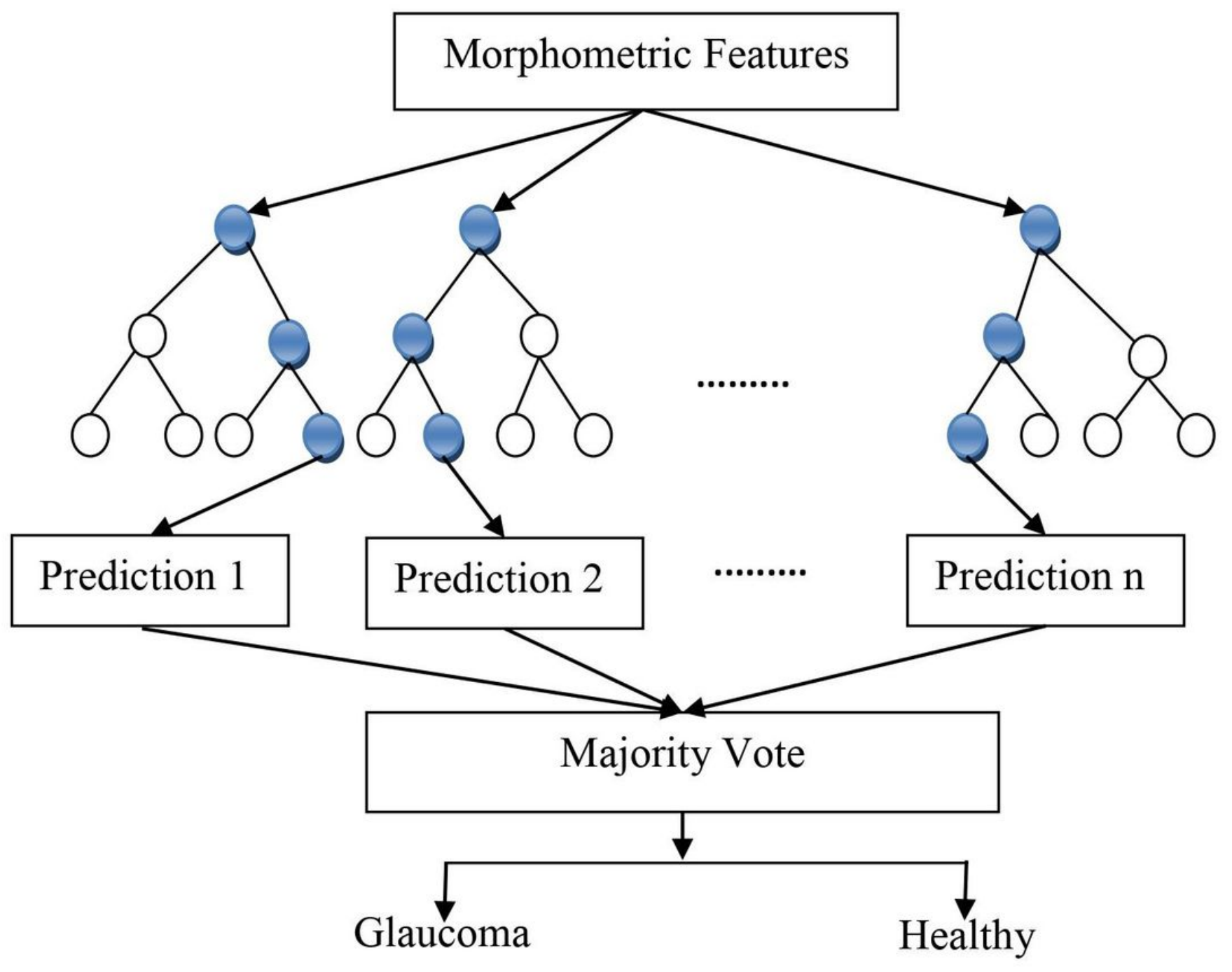

Figure 3

Random Forest Classification 

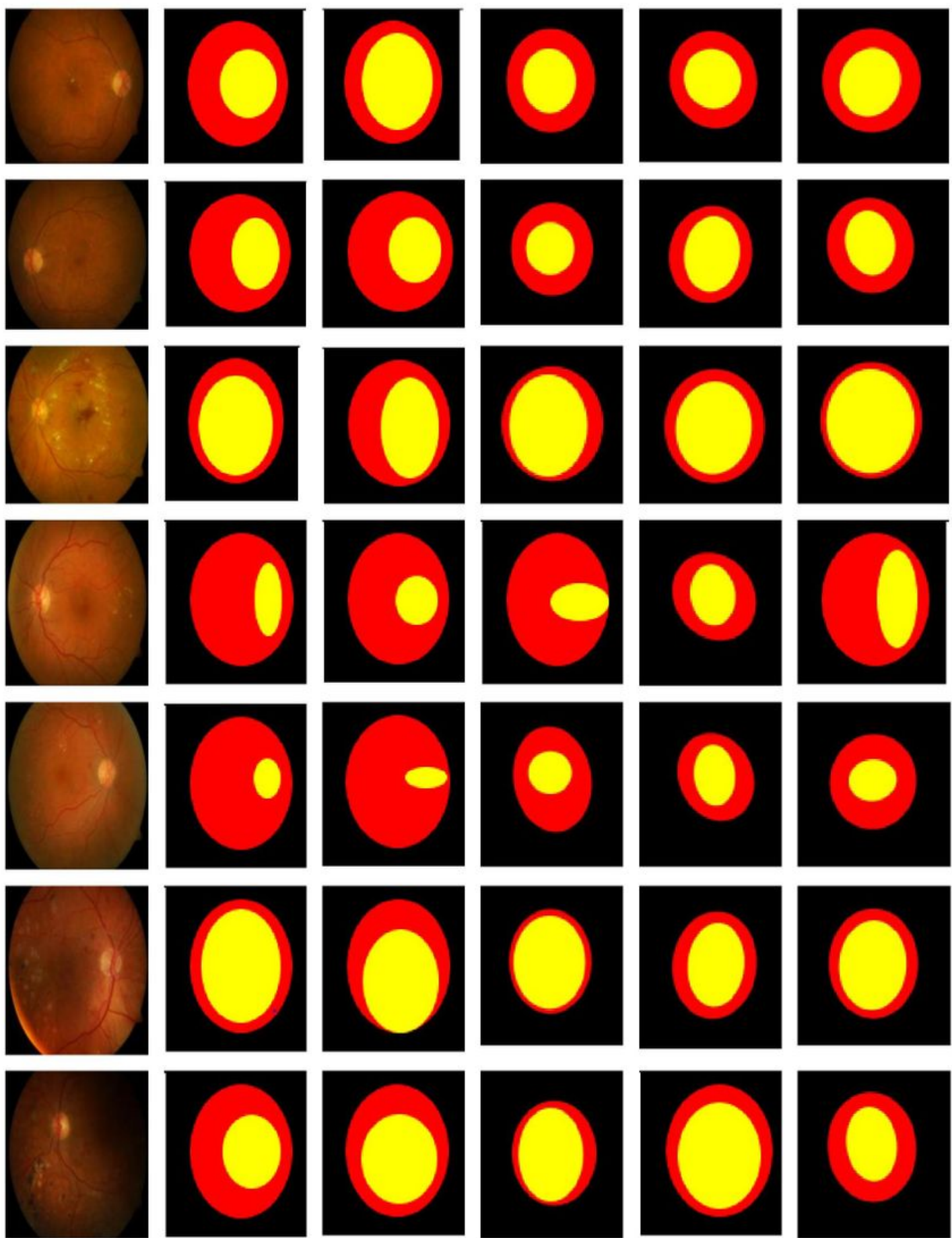

(a)

(b)

(c)

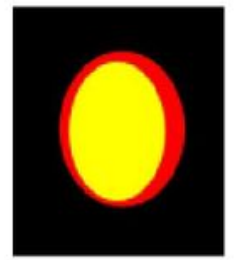

(d)

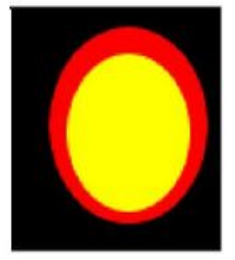

(e)

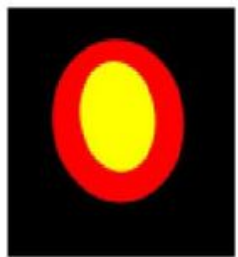

(f)

Figure 4

Simulation results a) original fundus image b) Original U-Net c) Full-Deformable U-Net d) Deformable UNet e) Au-Net and g) proposed method 


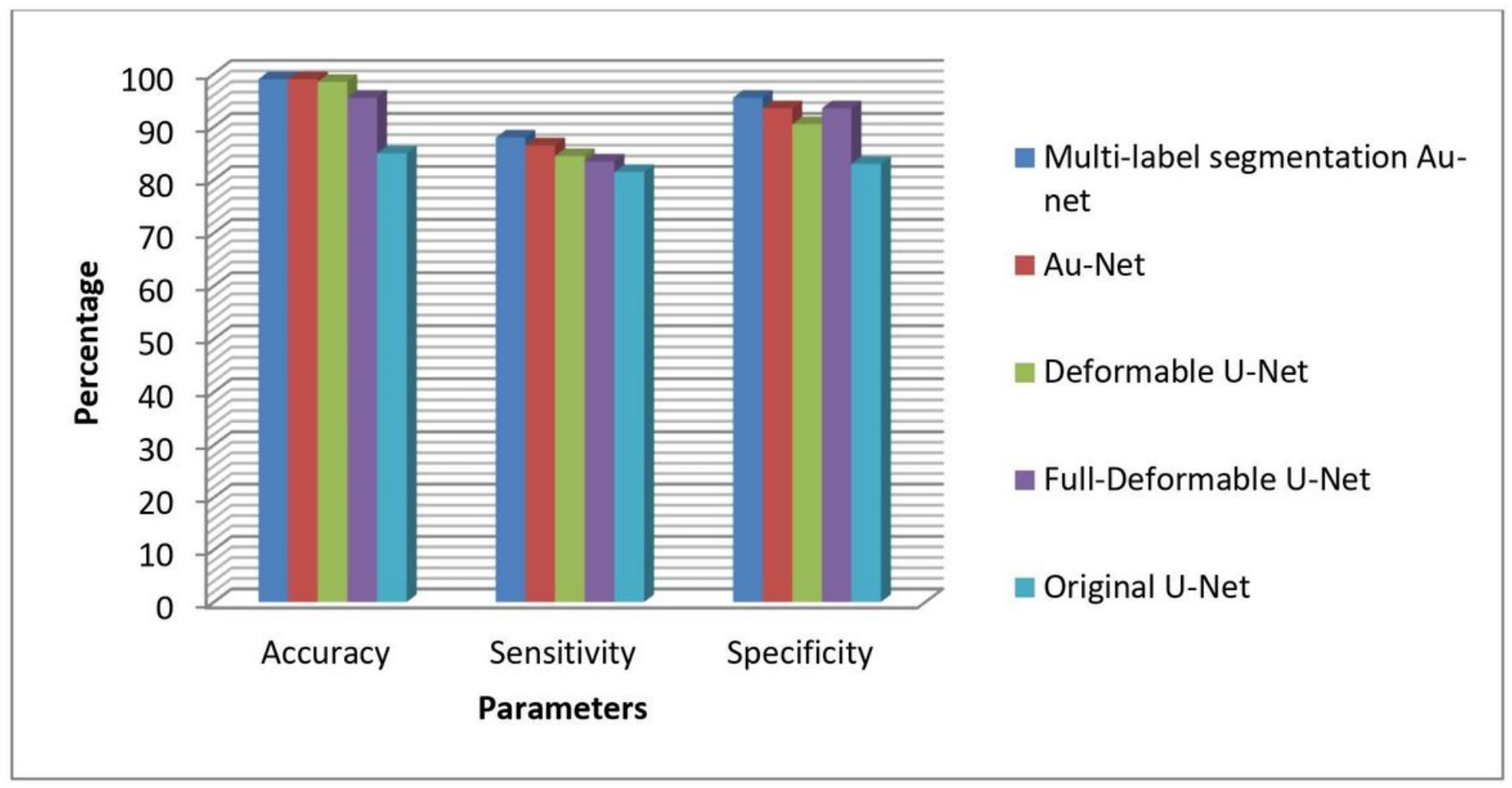

\section{Figure 5}

Comparative analysis of Proposed method with different segmentation methodoogies 


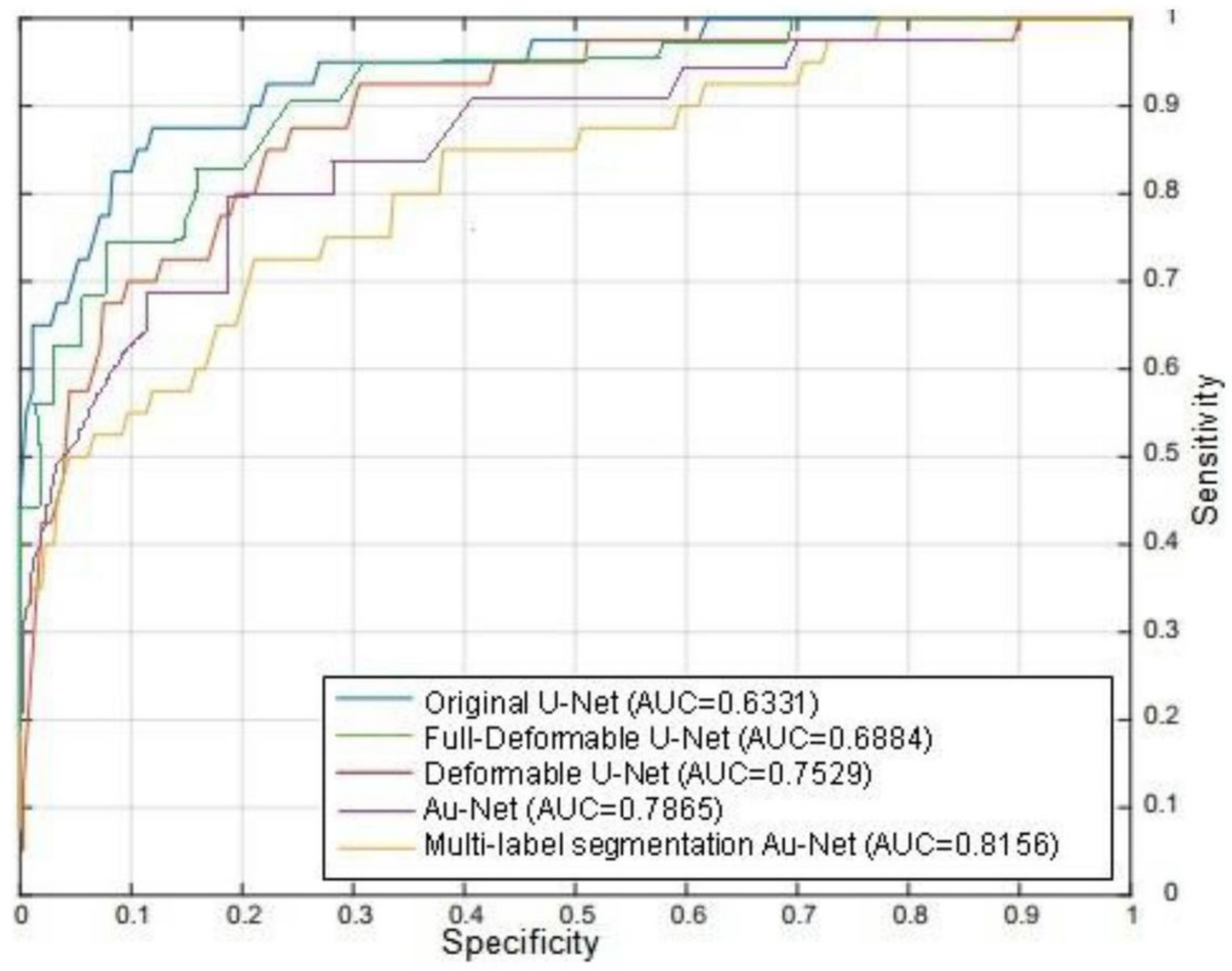

Figure 6

This paper additionally compute the intraocular pressure and the simulation outcomes are shown in Fig 6. 